\title{
Influence of Xiaoyaosan on depressive-like behaviors in chronic stress-depressed rats through regulating tryptophan metabolism in hippocampus
}

This article was published in the following Dove Press journal:

Neuropsychiatric Disease and Treatment

\section{Haiyan Jiao ${ }^{1, *}$ \\ Zhiyi Yan ${ }^{1, *}$ \\ Qingyu $\mathrm{Ma}^{2}$ \\ Xiaojuan $\mathrm{Li}^{2}$ \\ Youming Jiang' \\ Yueyun Liu' \\ Jiaxu Chen ${ }^{1,2}$}

'School of Traditional Chinese Medicine, Beijing University of Chinese Medicine, Beijing 100029 ,

China; ${ }^{2}$ Formula-Pattern Research Center, School of Traditional Chinese Medicine, Jinan University, Guangzhou 5I0632, Guangdong, China

*These authors contributed equally to this work
Correspondence: Jiaxu Chen School of Traditional Chinese Medicine, Beijing University of Chinese Medicine, II Northern Third Ring Road East, Chaoyang District, Beijing 100029, China Tel +86106428 6656

Email chenjiaxu@hotmail.com
Background: Tryptophan metabolism has always been considered to play a vital role in mental disorder diseases, and how traditional Chinese formula Xiaoyaosan regulates the tryptophan metabolism is a complement to the pathogenesis of depression. This study established a depression rat model by the chronic immobilization stress (CIS) method and observed the change in tryptophan metabolism in hippocampus and the effects of Xiaoyaosan.

Methods: Forty-eight male Sprague Dawley (SD) rats were randomly divided into the following four groups: control group, CIS group, Xiaoyaosan group, and fluoxetine group. The depression model was established by the 21-day CIS. The food intake and body weight were recorded, and the sucrose preference test (SPT), novelty suppressed feeding (NSF) test and open field test (OFT) were also used to evaluate the model. Then, the contents of tryptophan and 5-hydroxytryptamine (5-HT) in hippocampus were detected by the ELISA method, and the expression levels of tryptophan hydrogenase 2 (TPH2) and indoleamine 2,3-dioxygenase 1 (IDO1) in hippocampus were determined by quantitative reverse transcriptase polymerase chain reaction reaction (qRT-PCR) and Western blot methods.

Results: The behavioral data showed a significant difference between the model group and the normal group. The 5-HT content in the hippocampi of CIS rats was significantly reduced, whereas the tryptophan content in the hippocampi of model rats was significantly increased. The TPH2 level in hippocampus of the model group was significantly decreased, and the IDO1 level was significantly increased. Xiaoyaosan and fluoxetine could significantly reverse these changes and had obvious curative effects.

Conclusion: The abnormal tryptophan metabolism existed in the hippocampi of chronic stressdepressed rats, which was closely related to the pathogenesis of depression. Xiaoyaosan could improve the tryptophan metabolism by regulating the expression levels of TPH2 and IDO1, thus exerting an antidepressant-like effect.

Keywords: Xiaoyaosan, depression, chronic immobilization stress, tryptophan, TPH2, IDO1, traditional Chinese formula, traditional Chinese medicine

\section{Introduction}

Depression is a prevalent chronic mental disorder with high rates of recurrence, disability, and suicide, and it is often characterized by significant and persistent depressive disorder. ${ }^{1}$ Nowadays, along with the acceleration and change in lifestyle, the stress increases due to many aspects such as employment, social competition, and interpersonal communication, which leads to a gradual increase in the incidence of depression. 
According to WHO, depression has become one of the major causes of disability in the world, and it may become one of the main causes of the increased burden of disease and medical cost by $2030{ }^{2,3}$ Monoamine neurotransmitter hypothesis is one of the core concepts of depressive disorder caused by stress. The hypothesis suggests that the reduction or absence of 5-hydroxytryptamine (5-HT), norepinephrine (NE) or other monoamine neurotransmitters in the central and peripheral regions may cause the depression. ${ }^{4,5}$

5-HT, the most important intermediate metabolite in the tryptophan metabolism, is composed of indole and ethylamine. The decrease in 5-HT level in the central nervous system will enhance the sensitivity of the body to stress and make it more vulnerable to external injury, which leads to the depression or other mental disorders. ${ }^{6}$ A study has shown that the dysfunction of the 5-HT system is closely related to depression, anorexia, insomnia, circadian rhythm disorder, endocrine dysfunction, sexual dysfunction, anxiety disorder and reduced activity. ${ }^{7}$ Tryptophan hydrogenase (TPH) is the only ratelimiting enzyme of the 5-HT synthesis pathway, which is an important prerequisite for 5-HT synthesis and may be used as a specific marker of 5-HT neurons. ${ }^{8}$ Tryptophan hydrogenase 2 (TPH2) is a subtype of TPH that mainly controls the synthesis of central 5-HT, the abnormal expression of which may result in affective disorder and suicidal behavior. ${ }^{9}$ Indoleamine 2,3-dioxygenase 1 (IDO1) is a tryptophan-degrading enzyme whose increased activity may lead to increase in the formation of kynurenine due to tryptophan metabolism, resulting in the decrease in 5-HT synthesis. ${ }^{10}$ Therefore, the abnormal expression of TPH2 and IDO1 may cause the dysfunction of the 5-HT system, leading to the occurrence of mental disorders such as depression. Hippocampus is rich in various neurotransmitters and receptors and is closely related to emotion. It is the highregulation center of the stress response, and its structure and function could be changed by chronic stress. ${ }^{11}$ Hippocampus is also a target of stress hormones, and stress and high glucocorticoids can affect the hippocampal neuroplasticity. ${ }^{12}$ It is reported that $\mathrm{TPH} 2$ gene is expressed in the hippocampus and raphe nuclei, ${ }^{13}$ and IDO1 has a certain effect on the function and structure of hippocampal neurons. ${ }^{14}$ Therefore, hippocampus may be a key region to observe the stress-induced changes in TPH2 and IDO1 in the 5-HT system.

Xiaoyaosan, a classic Chinese formula in the book Prescriptions of the Bureau of Taiping People's Welfare Pharmacy, originates from the Song Dynasty of China. It has the effects of dispersing stagnated liver qi for relieving qi stagnation, nourishing blood and strengthening spleen. ${ }^{15}$ Xiaoyaosan has obvious comprehensive efficacy in treating depression caused by stress and other complex psychiatric disorders. ${ }^{16}$ Hippocampus is the most important area of the brain responsible for learning, memory and emotion regulation, and the malfunction of hippocampus could directly lead to the decline in the ability of learning, memory and emotional control. ${ }^{17}$ It is found that Xiaoyaosan has a certain regulatory effect on the structure and function of hippocampus, and hippocampus may play a key role in the antidepressant efficacy of Xiaoyaosan. ${ }^{18,19}$ One recent study indicates that the therapeutic effects of Xiaoyaosan may be associated with the function of the 5-HT system, ${ }^{20}$ but how Xiaoyaosan affects the synthesis of 5-HT through the tryptophan metabolism is still not understood.

Therefore, we used chronic immobilization stress (CIS) to establish the depression model of rats, and then we used ELISA to detect the levels of 5-HT and tryptophan and Western blot and quantitative reverse transcriptase polymerase chain reaction (qRT-PCR) to measure the expression of TPH2 and IDO1, in order to assess the influence of Xiaoyaosan on the tryptophan metabolism, which is responsible for the increase in the synthesis and function of 5-HT in the hippocampi of chronic stress-depressed rats.

\section{Materials and methods}

\section{Animals}

The specific pathogen-free (SPF) healthy male Sprague Dawley (SD) rats (12-week old; SCXK (Beijing) 2012-0001) were purchased from Beijing Vital River Laboratory Animal Technology Co., Ltd. (Beijing, China) and then fed in a standard animal feeding room (temperature: $20^{\circ} \mathrm{C}-24^{\circ} \mathrm{C}$; relative humidity: $30 \%-40 \%$ and light condition: 12 -hour/12-hour dark/light cycle for all the experiments).

All animal experiments in the study were approved by the Institutional Animal Care and Use Committee at Beijing University of Chinese Medicine and complied with the Animal Management Rules of the Chinese Ministry of Health and existing current animal welfare guidelines.

\section{Preparation of Xiaoyaosan}

The compound used in the experiment is Xiaoyaosan, which contains the following eight Chinese herbs: Radix Angelicae Sinensis, Radix Paeoniae Alba, Poria, Radix Bupleuri, Rhizoma Atractylodis Macrocephalae, Radix Glycyrrhizae, Herba Menthae and Rhizoma Zingiberis Recens (ratio: 3:3:3:3:3:1.5:1:1). These herbs were purchased from Beijing Tongrentang Group Co., Ltd. (Beijing, China). and extracted in a Chinese medicine preparation room of China-Japan Friendship Hospital according to a previous 
study. ${ }^{21}$ The extraction rate was $18.8 \%$, and the HPLC-mass spectrometry analysis (LC-MS/MS) was used to identify the eight compounds of Xiaoyaosan samples. ${ }^{16}$

\section{CIS and medication process}

A total of 48 rats were randomly divided into the following four groups according to their body weights: control group (no stress with physiological saline, $n=12$ ), CIS group (CIS with physiological saline, $n=12$ ), Xiaoyaosan group (CIS with Xiaoyaosan treatment, $n=12$ ) and fluoxetine group (CIS with fluoxetine treatment, $\mathrm{n}=12$ ). The CIS procedure is shown in Figure 1 and performed as previously described. ${ }^{22}$ Briefly, the rats in the CIS, Xiaoyaosan and fluoxetine groups were exposed to a homemade apparatus, which was used to immobilize rats. This apparatus was composed of wooden double-binding platforms, including a lower platform $(20 \times 10 \times 2.8 \mathrm{~cm})$ and an upper platform $(22 \times 6.6 \times 2.8 \mathrm{~cm})$. In front of the upper platform, a small frame suitable was located for immobilizing a rat head. Meanwhile, two pieces of soft, adjustable bands were used to fix the upper body and waist of a rat on the upper platform. The rats were immobilized in this apparatus for 3 hours a day, without free access to food or water, and CIS was performed for successive 21 days. Rats in the two treatment groups received Xiaoyaosan $(3.854 \mathrm{~g} / \mathrm{kg} / \mathrm{d}, 0.1 \mathrm{~mL} / \mathrm{kg}$ bodyweight) and fluoxetine $(2 \mathrm{mg} / \mathrm{kg} / \mathrm{d}, 0.1 \mathrm{~mL} / \mathrm{kg}$ bodyweight $)$ by intragastric administration after the daily immobilization procedure, respectively. Meanwhile, rats in the control and CIS groups received physiological saline $(0.1 \mathrm{~mL} / \mathrm{kg}$ bodyweight $)$. Furthermore, to observe the behaviors of the rats during the CIS process, the body weight and food intake were recorded weekly (days $0,7,14$ and 21), and the data of the day before experiment were used as baseline (day 0). The other behavioral assessments such as OFT (days 0 and 21),
SPT (days 0 and 21) and NSF (day 21) were performed before or after the CIS procedure.

\section{Sucrose preference test (SPT)}

The SPT was carried out before and after the stress period (days 0 and 21), and it was performed as described in a previous study. ${ }^{23}$ In short, each rat received two bottles of $1 \%$ sucrose solution for 24 hours; then one filled with $1 \%$ sucrose solution and the other with pure water for another 24 hours. After fasting and water deprivation for 24 hours, each rat received two bottles filled with pure water and 1\% sucrose solution for 1 hour. The ratio of consumption of sucrose solution to that of total solution within 1 hour was recorded, which could be the objective indicator of hedonic behavior.

\section{Novelty suppressed feeding (NSF) test}

The NSF test was conducted at day 21 using the previously described method. ${ }^{24}$ Briefly, a white plastic box was prepared $(50 \times 50 \times 40 \mathrm{~cm})$ with a single pellet of regular chow placed in the center. After food deprivation for 24 hours, the rats were individually placed in the corner of the plastic box. The time that the rats began to chew the food pellet was recorded up to 5 minutes as the feeding latency in the novelty environment. The box was cleaned, and the food pellet was replaced after each test.

\section{Open field test (OFT)}

The OFT was performed before and after the CIS process (days 0 and 21) based on the previously described procedure. ${ }^{25}$ A wooden box with open bottom $(125 \times 125 \times 50 \mathrm{~cm})$ was prepared, and the test was performed in a light- and sound-attenuated room with a dim light. The movement condition of rats in each group was recorded for 5 minutes

CIS drug administration

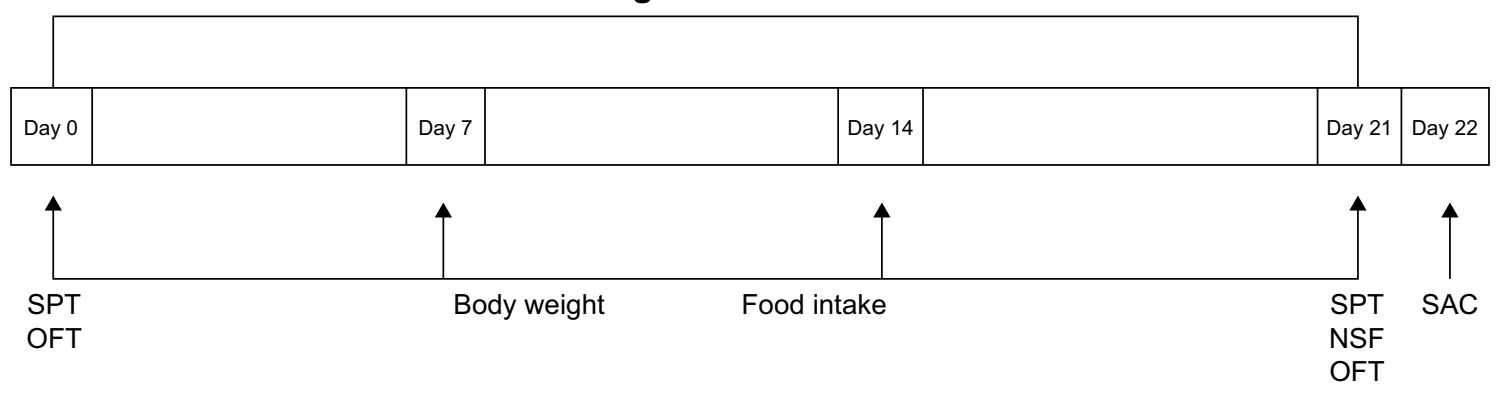

Figure I Study schedule.

Notes: CIS and drug administration were conducted for successive 21 days; the body weight and food intake were recorded at days 0,7 , I4 and 21 ; SPT and OFT were performed at day 0, and then SPT, NSF and OFT were performed at day 2I; rats were sacrificed at day 22.

Abbreviations: CIS, chronic immobilization stress; NSF, novelty suppressed feeding test; OFT, open field test; SAC, sacrificed; SPT, sucrose preference test. 
by an HD camera. The Etho Vision 3.0 software (Noldus, Wageningen, Holland) was used to record the locomotor ability of each rat (lattice number, residence time and number of entries into central area).

\section{Preparation of tissues}

After 21 days of CIS modeling, all rats in four groups were anesthetized by an intraperitoneal injection of $10 \%$ chloral hydrate. The hippocampal tissues of six rats in each group were collected for protein analysis (Western blot and ELISA analyses), and then hypothalamic tissues of the other six rats in each group were collected with RNA preservation solution (Biotech, \#2714) for qRT-PCR assay.

\section{qRT-PCR analysis}

The levels of TPH2 and IDO1 mRNAs in the hippocampus were evaluated by qRT-PCR assay. The total RNA of hypothalamus was extracted using Trizol reagent (Thermo Fisher Scientific, Waltham, MA, USA). Then, the concentration and quality of total RNA were determined by Q3000 micro-volume spectrophotometer (Quawell Technology, San Jose, CA, USA) and 1\% agarose gel electrophoresis. The RevertAid First Strand cDNA Synthesis Kit (Thermo Fisher Scientific) was used to synthesize first-strand cDNA on C1000 Touch TM Thermal Cycler (Bio-Rad Laboratories Inc., Hercules, CA, USA) according to the standard protocol. The SYBR ${ }^{\circledR}$ Green PCR Master Mix (Thermo Fisher Scientific) was used to amplify the cDNA in the Multicolor Real-time PCR Detection System (Bio-Rad Laboratories Inc.), and the cycling parameters were as follows: $95^{\circ} \mathrm{C}$ for 10 minutes, followed by 40 cycles of $95^{\circ} \mathrm{C}$ for 15 seconds and $56^{\circ} \mathrm{C}(\mathrm{TPH} 2)$ or $58^{\circ} \mathrm{C}$ (IDO1) for 1 minute, then followed by $65^{\circ} \mathrm{C}$ for 5 seconds and $95^{\circ} \mathrm{C}$ for 15 seconds. The $2^{-\Delta \Delta \mathrm{Ct}}$ method was used to calculate the expression of mRNA. Table 1 shows the sequences for primers, which were designed according to the published mRNA sequences in NCBI, and GAPDH (BBI Life Science, Amherst, MA, USA) was used as the house-keeping gene in the experiment.

Table I Primer sequences used in the qRT-PCR analysis

\begin{tabular}{l|l}
\hline Gene & Sequences \\
\hline TPH2 & \\
Forward & 5'-CCATCGGAGAATTGAAGCAT-3' \\
Reverse & 5'-TTGGAAGGTGGTGATTAGGC-3' \\
IDOI & \\
Forward & 5'-GGGCTTTGCTCTACCACATC-3' \\
Reverse & 5'-GCTTCCCATTCTCAATCAGC-3' \\
\hline
\end{tabular}

Abbreviations: IDOI, indoleamine 2,3-dioxygenase I; qRT-PCR, quantificational real-time polymerase chain reaction; TPH2, tryptophan hydrogenase 2 .

\section{Western blot analysis}

The expression of the TPH2 and IDO1 proteins in the hippocampus was detected by Western blot. The hippocampal tissues were used to prepare the total proteins with RIPA Lysis buffer (Biomiga, Santiago, CA, USA). The 10\% SDSPAGE gels were selected according to relative molecular weight of TPH2 (56 kDa) and IDO1 (45 kDa), and then proteins were transferred onto polyvinylidene fluoride (PVDF) membranes. The 5\% nonfat milk was used to block the membranes, and the primary antibodies were anti-TPH2 antibody (ab121013, goat polyclonal to TPH2, diluted 1:500; Abcam, San Francisco, CA, USA), anti-indoleamine 2,3-dioxygenase antibody (ab106134, rabbit polyclonal to indoleamine 2,3-dioxygenase, diluted 1:50; Abcam) and $\beta$-actin monoclonal antibody (MA1-140, mouse monoclonal to $\beta$-actin, diluted 1:3,000; Thermo Fisher Scientific). The enhanced chemiluminescence (ECL) detection reagent (Thermo Fisher Scientific) was used to develop the membranes for 1 minute, and then the Tanon-5200 system (Tanon, Shanghai, China) was used for exposure. The optical density of protein bands was read by Tanon Gis software (Tanon).

\section{ELISA analysis}

The weight of each hippocampal tissue was recorded, and then pulverized with pre-cooling PBS $(0.01 \mathrm{M}, \mathrm{pH} 7.4)$ in a certain ratio (tissue weight: PBS volume $=1 \mathrm{mg}: 9 \mu \mathrm{L}$ ) and centrifuged to prepare the diluted samples. The contents of 5-HT and tryptophan were measured by ELISA assay (Serotonin ELISA kit, ADI-900-175; Enzo, New York, NY, USA and Tryptophan ELISA kit, KA1916; Abnova, Taipei City, China) according to the standard protocol of the kits. The mean optical density was detected using the Multiskan ${ }^{\text {TM }} \mathrm{GO}$ (Thermo Fisher Scientific) Detector system at a wavelength of $405 \mathrm{~nm}$ (5-HT) or $450 \mathrm{~nm}$ (tryptophan), and the content was calculated according to the standard curves and relative regression equations.

\section{Statistical methods}

All data were expressed as mean \pm standard error of the mean (SEM) and analyzed by the SPSS 21.0 software, one-way ANOVA or nonparametric test was performed for general data based on the normality test and the homogeneity test for variance, and the least significant difference (LSD) method was used for post hoc comparisons when equal variances were assumed, whereas Dunnett's T3 test was used when the data had a normal distribution but the variances are not homogeneous. In addition, repeated measurement process of general linear model (GLM) was measured to conduct 
one-way ANOVA for repeated measured data such as body weight and food intake. A $P$-value of $<0.05$ was considered statistically significant, and the GraphPad Prism 6.0 software was used to draw statistical graphs.

\section{Results}

\section{Xiaoyaosan improved food intake and body weight of chronic stress- depressed rats}

In order to evaluate the efficacy of Xiaoyaosan to the physical condition of CIS-exposed rats, the data of food intake and body weight were recorded and analyzed. Figure 2 shows that the body weight and food intake of rats in each group had no significant differences at day 0 , but difference appeared gradually as the CIS procedure continued. On day 21, the body weight and food intake between the CIS group and the control group were significantly different (body weight: $\mathrm{f}(3,44)=4.685$, food intake: $\mathrm{f}(3,44)=4.025$, both $P<0.01)$, while the treatment of both Xiaoyaosan and fluoxetine could significantly improve the food intake and body weight compared with the CIS group (both $P<0.01$ ).

\section{Xiaoyaosan improved depressive-like behaviors of chronic stress-depressed rats}

Several behavioral assessments were carried out to further evaluate the efficacy of Xiaoyaosan to depressive-like behaviors of CIS-exposed rats. As shown in the SPT results, each group had a similar sucrose preference baseline at day 0 $(f(3,44)=4.163, P>0.05$; Figure $3 \mathrm{~A})$, and after modeling for 21 days, the sucrose preference of rats in the CIS group significantly decreased compared with the rats in the control group

A

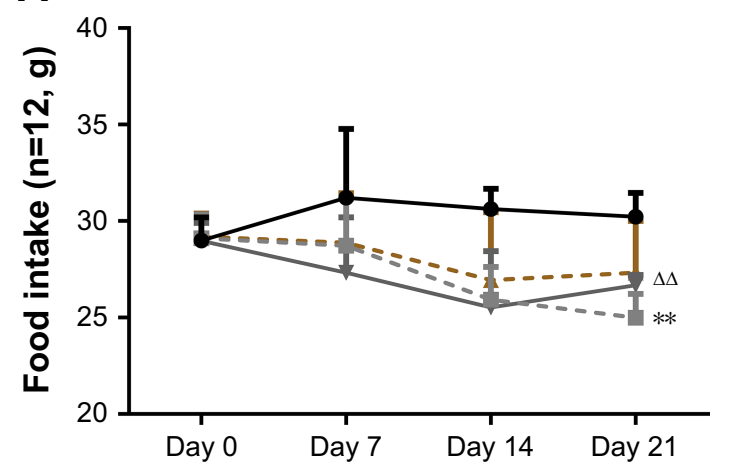

(f(3, 44) $=4.928, P<0.01$; Figure 3B); Xiaoyaosan or fluoxetine treatment could significantly reverse the reduction of sucrose preference (both $P<0.01$ ). As shown in the NSF test, the latency to eat the chow represented the degree of anxiety/ depression in the CIS-exposed rats. It was shown that CIS rats had a longer latency to chew the chow than the control rats and had a significant difference $(\mathrm{f}(3,44)=4.129, P<0.01$; Figure $3 \mathrm{C}$ ). The latencies of rats in two treatment groups were significantly lower than those of the CIS rats (both $P<0.05$ ). Figure 3D shows a representative moving trail of rats in each group at day 21. The OFT results showed that baselines of all the groups had no obvious difference at day 0 (number of entries into the central area: $f(3,44)=2.096$, residence time: $\mathrm{f}(3,44)=0.571$, lattice number: $\mathrm{f}(3,44)=0.876$, both $P>0.05$; Figure $3 \mathrm{E}, \mathrm{G}$ and I). After modeling for 21 days, the behaviors of the CIS-exposed rats were significantly different from the control rats (number of entries into the central area: $\mathrm{f}(3,44)=2.113$, residence time: $\mathrm{f}(3,44)=4.017$, lattice number: $\mathrm{f}(3,44)=4.366$, both $P<0.01$; Figure $3 \mathrm{~F}, \mathrm{H}$ and $\mathrm{J})$. Both Xiaoyaosan and fluoxetine treatments could remarkably reverse these depressive-related behavioral changes $(P<0.05$ and $P<0.01$, respectively).

\section{Xiaoyaosan improved the tryptophan metabolism in the hippocampi of chronic stress-depressed rats}

In order to observe the mechanisms that Xiaoyaosan regulated the tryptophan metabolism in the hippocampi of chronic stress-depressed rats, the expression levels of TPH2 and IDO1 were measured, while the contents of 5-HT and tryptophan were detected. The mRNA and protein expression results

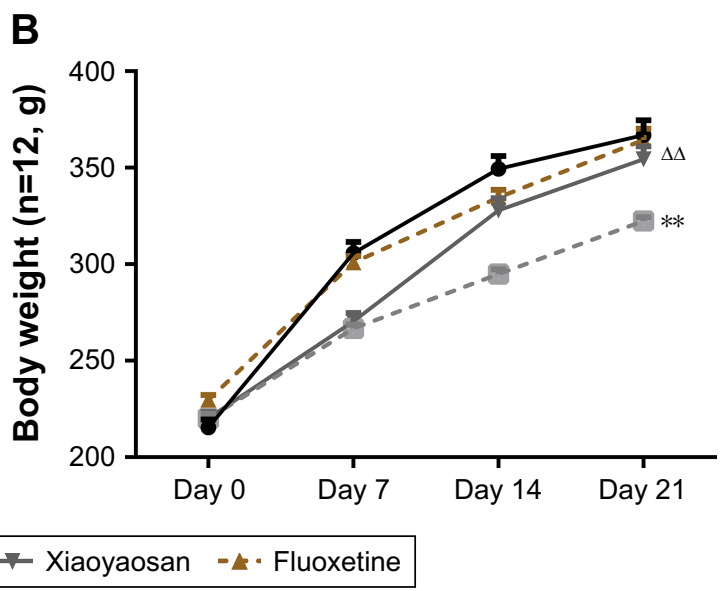

Figure 2 Changes in the food intake and body weight of chronic stress-depressed rats.

Notes: (A) Changes in food intake during the modeling period $(n=12)$. (B) Changes in body weight during the modeling period ( $n=12)$. Data are expressed as mean \pm SEM, $* * P<0.0$ I vs the control group; ${ }^{\Delta} P<0.01$ vs the CIS group.

Abbreviations: CIS, chronic immobilization stress; SEM, standard error of the mean. 

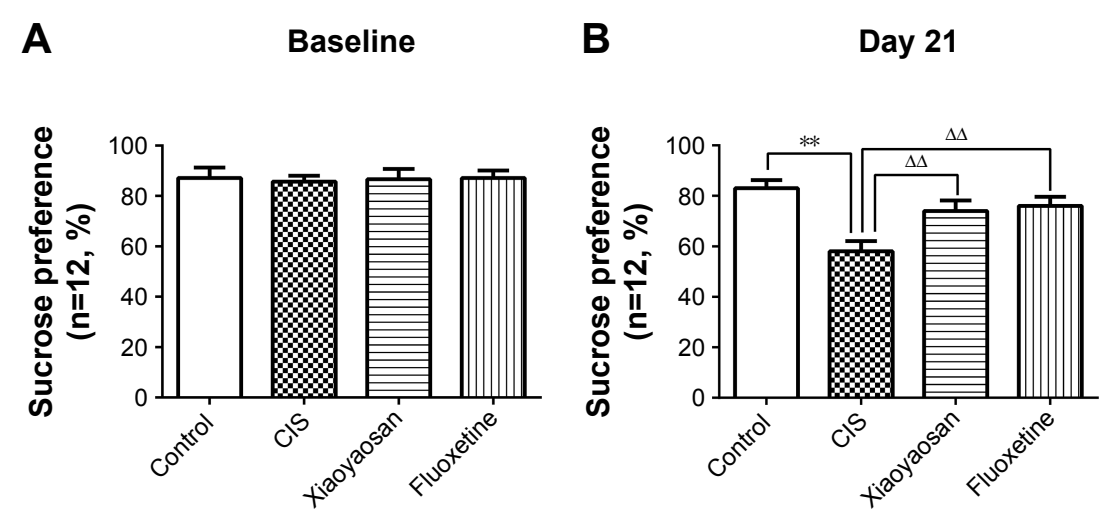

C NSF

D

Control

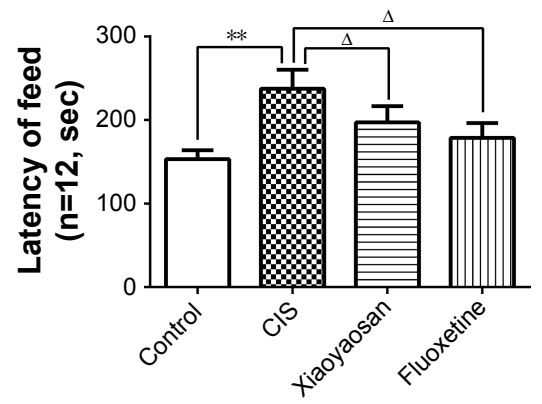

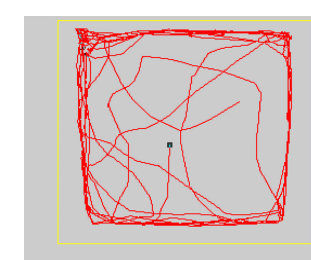

E

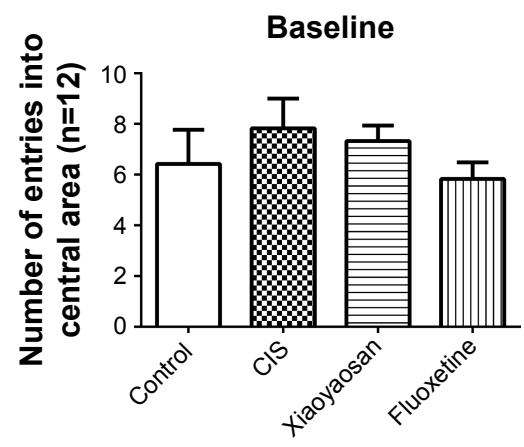

F

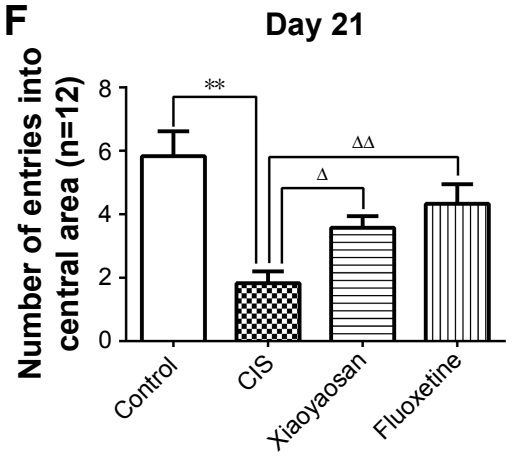

CIS

Xiaoyaosan

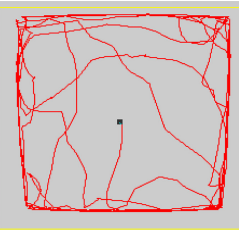

G

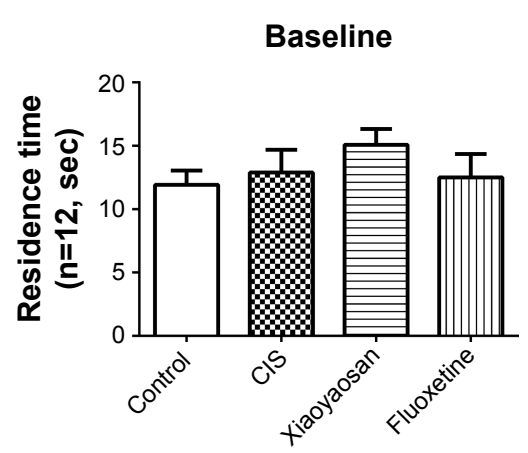

H

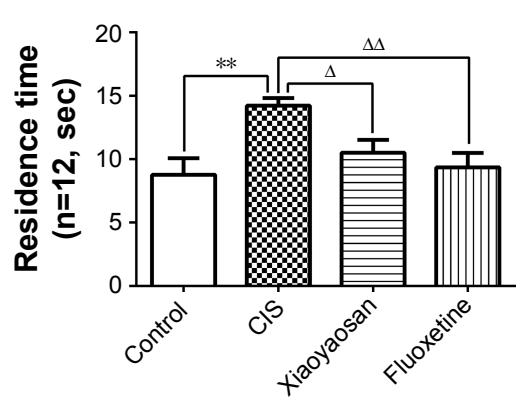

\section{Fluoxetine}

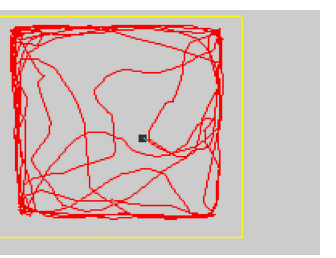

I

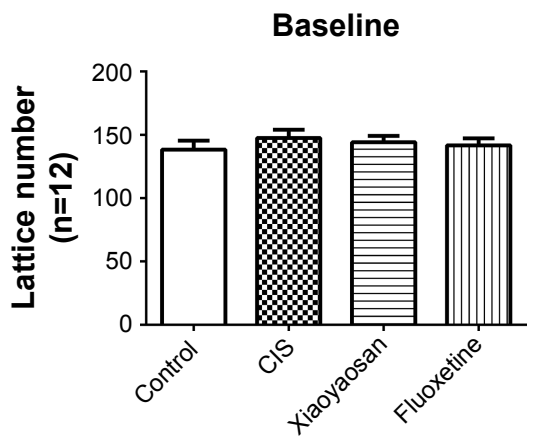

J

Day 21

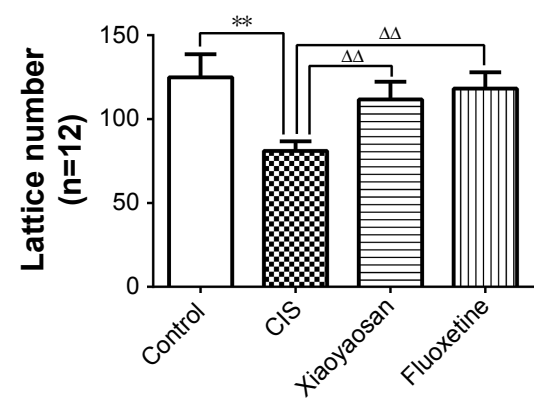

Figure 3 Changes in the depressive-like behaviors of chronic stress-depressed rats.

Notes: (A) SPT results at day 0 (baseline, $n=12$ ); (B) SPT results at day $2 \mid(n=12)$; and (C) NSF results at day 21 ( $n=\mid 2)$. (D) The representative moving trails of rats in each group at day $2 \mathrm{I}$ assessed by video tracking software. (E) The results of number of entries into the central area at day 0 (baseline, $\mathrm{n}=12$ ). (F) The results of number of entries into the central area at day $2 \mid(n=\mid 2)$. (G) The results of residence time at day 0 (baseline, $n=\mid 2)$. (H) The results of residence time at day $2 \mid$ ( $n=\mid 2)$. (I) The results of lattice number at day 0 (baseline, $n=\mid 2)$. (J) The results of lattice number at day $2 I(n=\mid 2)$. Data were expressed as mean $\pm S E M$, $* * P<0.0 I$ vs the control group; ${ }^{\Delta P}<0.05$, $\triangle \triangle P<0.01$ vs the CIS group.

Abbreviations: CIS, chronic immobilization stress; NSF, novelty suppressed feeding; SEM, standard error of the mean; sec, seconds; SPT, sucrose preference test.

revealed that the 21-day CIS decreased the TPH2 levels in the hypothalamus of chronic stress-depressed rats (TPH2 mRNA: $\mathrm{f}(3,20)=6.643$, TPH $2 / \beta$-actin: $\mathrm{f}(3,20)=5.003$, both $P<0.05$; Figure 4A and C), and the TPH2 levels of rats in the Xiaoyaosan and fluoxetine groups significantly increased compared with the CIS rats (both $P<0.05$ ). The 21-day CIS also increased the IDO1 levels in the hypothalamus (IDO1 mRNA: $\mathrm{f}(3,20)=19.170828$, IDO $1 / \beta$-actin: $\mathrm{f}(3,20)=1.466$, 
A
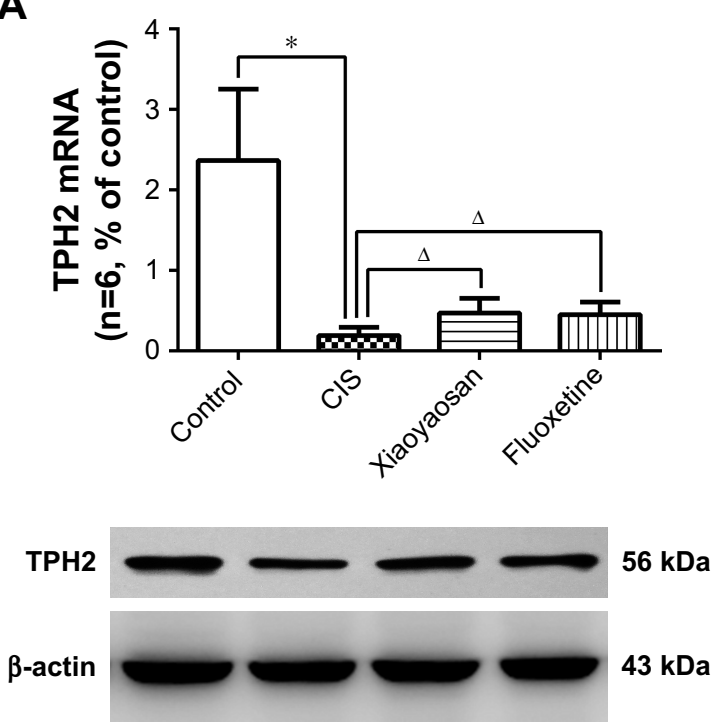

C

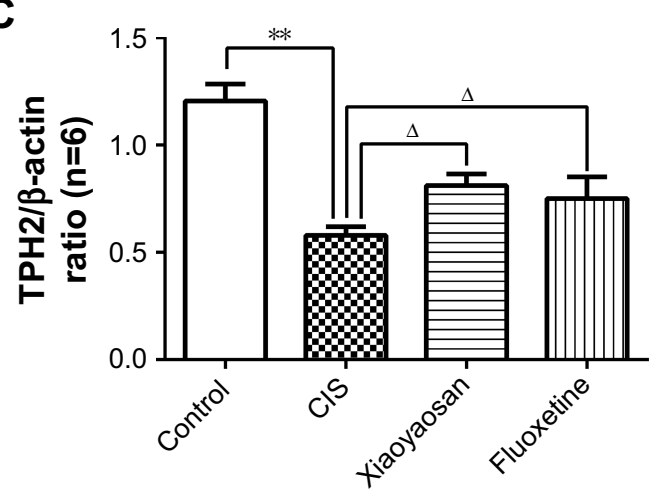

E

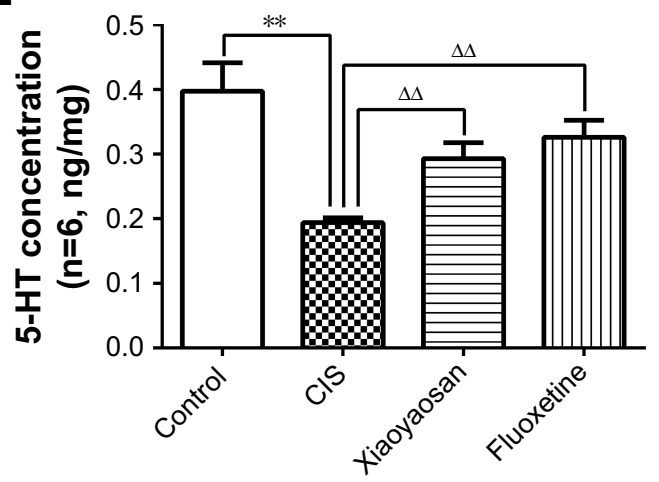

B
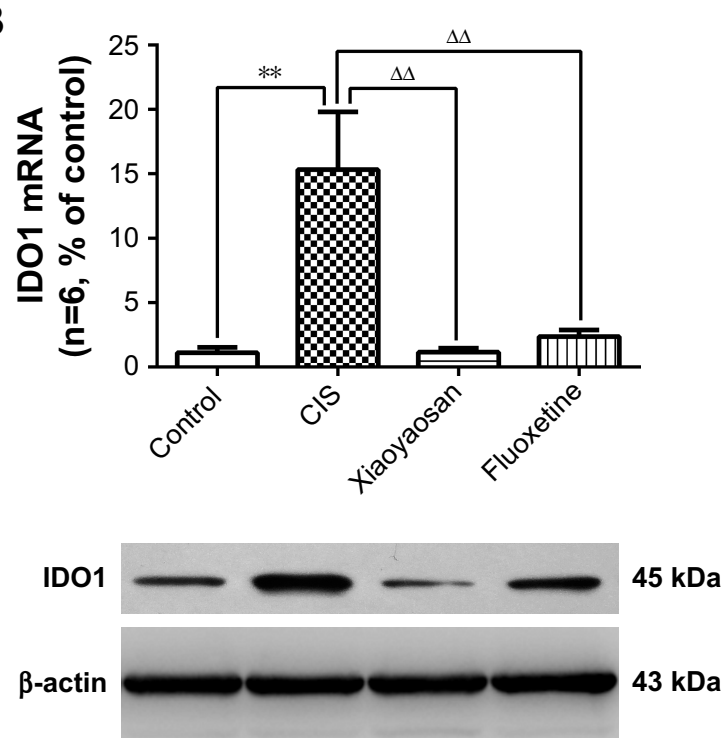

D

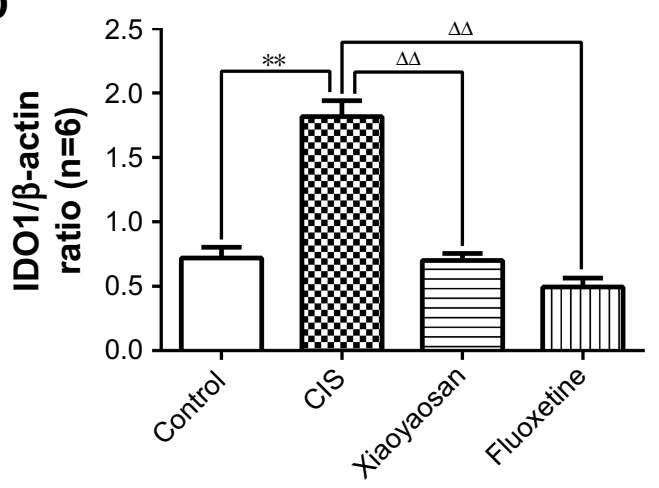

$\mathbf{F}$

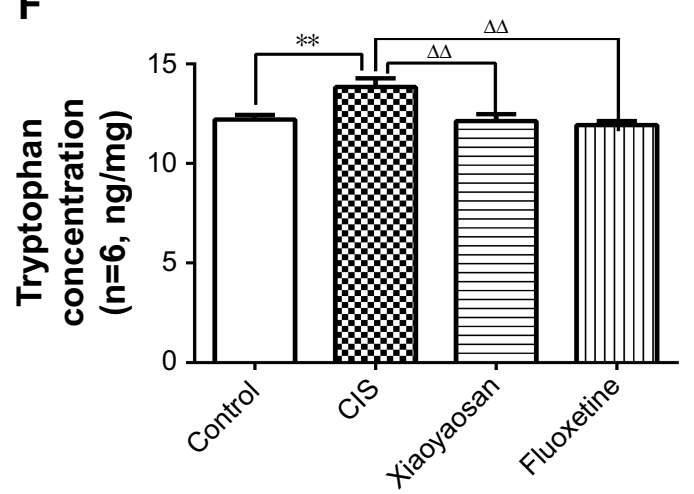

Figure 4 Changes in the hippocampal tryptophan metabolism of chronic stress-depressed rats.

Notes: (A) The mRNA results of TPH2 ( $n=6)$. (B) The mRNA results of IDOI $(n=6)$. (C) The protein results of TPH2 $(n=6)$. (D) The protein results of IDOI ( $n=6)$. (E) The results of $5-\mathrm{HT}$ contents $(n=6)$. (F) The results of tryptophan contents $(n=6)$. Data were expressed as mean $\pm S E M, * P<0.05, * * P<0.0$ I vs the control group; ${ }^{\Delta} P<0.05$, $\Delta \triangle P<0.01$ vs the CIS group.

Abbreviations: CIS, chronic immobilization stress; 5-HT, 5-hydroxytryptamine; IDOI, indoleamine 2,3-dioxygenase I; SEM, standard error of the mean; TPH2, tryptophan hydrogenase 2 .

both $P<0.01$; Figure 4B and D), both Xiaoyaosan and fluoxetine could significantly inhibit the increase in IDO1 level compared with the CIS group (both $P<0.01$ ).

The standard curves in this study were constructed by plotting the peak area of gradient concentrations of the two reference standards. The calibration curves were as follows: $\mathrm{y}=-0.129 \ln (\mathrm{x})+0.9349\left(\mathrm{R}^{2}=0.995,5-\mathrm{HT}\right)$ and $\mathrm{y}=-0.148 \ln (\mathrm{x})+1.0092\left(\mathrm{R}^{2}=0.993\right.$, tryptophan $)$. Compared with the control group, the contents of 5-HT in the hippocampi of CIS rats obviously decreased $(f(3,20)=4.076$, 
$P<0.01$; Figure 4E), whereas the content of tryptophan significantly increased $(\mathrm{f}(3,20)=3.181, P<0.01$; Figure $4 \mathrm{~F})$. The treatment of Xiaoyaosan or fluoxetine could remarkably reverse the changes in 5-HT and tryptophan contents $(P<0.05$ and $P<0.01$, respectively).

\section{Discussion}

The purpose of this study is to explore the pathogenesis of depression and the effect of Xiaoyaosan on the tryptophan metabolism, which was responsible for the increase in the synthesis and function of 5-HT. Immobilization stress, as a significant stress measure, has been widely applied in various animal experiments. ${ }^{26,27}$ The CIS-induced model, to a certain extent, simulates the state of depression in clinic. ${ }^{28,29}$ In this study, we found that Xiaoyaosan can alleviate the depressivelike behaviors in a rat model of depression built by the 21-day CIS. Our findings suggested that the treatment of Xiaoyaosan regulates the concentrations of 5-HT and tryptophan mainly by upregulating TPH 2 expression, as well as downregulating IDO1 expression in the hippocampi of depressed rats.

Depression, as a complex public health problem, has been widely studied for many years. But until now, it has become a major cause of medical disability worldwide and is a serious threat to human health. ${ }^{30}$ In order to reduce the detriment brought by depression, it is an effective way to determine the pathogenesis of depression by studying the mechanism of traditional Chinese medicine with antidepressant effect. Stress is a systemic non-specific adaptation reaction when the body is stimulated by various internal and external environmental factors, and it is closely related to the occurrence and development of many diseases. Mental disorders and cognitive impairment caused by stress are closely related to the limbic system and the important brain regions such as hypothalamus. ${ }^{31,32}$ Meanwhile, life stressor is an important inducing factor of clinical depression, thus stress is one of the important causes of mental disorders such as depression and anxiety. ${ }^{33}$

The SPT is a common method to observe the anhedonia induced by depression, and the decreased sucrose preference is a crucial indicator of depressive-like behaviors in rodents. ${ }^{34,35}$ As shown in our results, the differences in sucrose preference between control and CIS groups indicate the presence of obvious anhedonia and depression in the CIS rats. The NSF test was used to determine the animal's mental and behavioral changes by observing the feeding latency of animals in a novelty environment. ${ }^{36}$ The results revealed that the feeding latency of rats in the CIS group was significantly longer than that in the control group, which verified the depressive-like changes in the CIS rats. The OFT was carried out to evaluate the autonomous exploration of animals in a new environment and commonly used in the evaluation of animal models such as depression and anxiety. ${ }^{37,38}$ In this study, the lattice number, residence time and the number of entries into central area were analyzed. When the rats were in a state of anxiety or depression, it would take the initiative to avoid the open central area, choose to move around the box and reduce the number of entries into central area. Once entered into the open central area, the rats with depressive-like changes would be hesitant to move around, thus increasing the residence time. The lattice number mainly reflected that the total movement of experimental animals, the spontaneous activity and locomotor ability of rats decreased when exposed to CIS. According to the results, there were significant differences in the lattice number, residence time and the number of entries into central area in the CIS group compared with the control group, which further confirmed that CIS would cause depressive-like behaviors in the CIS group. Previous studies in clinical and animal experiments suggested that Xiaoyaosan had good relieving effects on depressive-like behaviors. ${ }^{39,40} \mathrm{We}$ had similar behavioral results in this study, wherein both Xiaoyaosan and fluoxetine treatments had a reasonable antidepressant-like effect according to the results of behavior tests, and fluoxetine was used as a positive control drug to verify the antidepressant-like effect of Xiaoyaosan. In addition, persistent stress and depression often led to low appetite and weight loss. ${ }^{41,42}$ During the CIS process, the difference in general states between the model group and the control group gradually became obvious, which confirmed the general manifestation of stress-induced depression. ${ }^{43}$ Our results indicated that Xiaoyaosan could effectively regulate appetite and the body weight of the CIS rats.

The decrease in 5-HT in the central nervous system could increase the body sensitivity to external stress and change the mental state, resulting in mental diseases such as depression and anxiety. ${ }^{44}$-HT was the most important intermediate product in the tryptophan metabolism. When the metabolism speed was accelerated due to stress, the level of tryptophan decreased, and then the content of 5-HT decreased and caused mental disorders. ${ }^{45}$ The results of this study showed a dysfunction of tryptophan metabolism in the CIS-induced rats, which is manifested by an increased level of tryptophan and a decreased level of 5-HT in the hippocampus. At the same time, the expression of TPH2 and IDO1 in the hippocampi of rats in the CIS group changed. CIS could cause an upregulation of the expression of IDO1 and a downregulation of the expression of $\mathrm{TPH} 2$, which 
suggested that CIS might inhibit the tryptophan metabolism to reduce the content of 5-HT in the hippocampi of rats. TPH 2 was abundant in the hippocampus and raphe nuclei; ${ }^{46}$ it mainly controlled the synthesis of 5-HT in the central nervous system. ${ }^{47}$ This meant that the decreased level of TPH2 may reduce the utilization rate of tryptophan and cause depression. ${ }^{48}$ The CIS may lead to a decrease in the utilization of tryptophan, followed by a decrease in 5-HT biosynthesis, which may be one of the important mechanisms of depression. IDO included two homologous proteins, IDO1 and IDO2, which were similar in structure and function. ${ }^{49}$ There was a positive correlation between the content of IDO and depressive-like symptoms. ${ }^{50}$ IDO1, one of the main indicators in this study, was closely related to monoamine neurotransmitters, cytokines and neuronal plasticity that played an important role in the pathogenesis and treatment of mental diseases. ${ }^{51}$ Excessive expression of IDO1 accelerated the consumption of 5-HT and produced more kynurenine from tryptophan metabolism. Tryptophan was an essential amino acid and a precursor substance of 5-HT. The decrease in tryptophan would lead to a decrease in 5 -HT in the absence of outside intake. ${ }^{52}$ In addition, some of the metabolites of the tryptophan-kynurenine pathway such as 3-hydroxykynurenine and quinolinic acid had neurotoxic effects under the action of IDO1, which might cause the degeneration of neurons and affect the neural plasticity. ${ }^{53,54}$ $\mathrm{TPH} 2$, as a key enzyme, directly regulated the synthesis of 5-HT, and IDO1 indirectly affected the 5-HT level by forcing the breakdown of tryptophan into kynurenine. Therefore, as shown in Figure 5, CIS could cause the abnormal expression of TPH2 and IDO1 in the hippocampus, which affected the tryptophan metabolism and the content of 5-HT. When the body was exposed to stress, tryptophan was overconsumed because of the excessive expression of IDO1. Meanwhile, the synthesis of 5-HT decreased due to tryptophan metabolism because of the downregulation of the expression of TPH2, which further increased the body's sensitivity to stress and then aggravated depressive symptoms.

As shown in the results, Xiaoyaosan and fluoxetine could regulate the expression of TPH 2 and IDO1 in the hippocampi of the rats exposed to CIS. The two drugs could allow more tryptophan to metabolize into 5-HT, had a certain recovery effect on the dysfunction of the 5-HT system and then played the antidepressant-like effect. A previous study had shown that fluoxetine has an upregulation effect on the expression of TPH2 in hippocampus, ${ }^{55}$ and fluoxetine could effectively inhibit the IDO activity to exert its anti-inflammatory effects, ${ }^{56}$ which was consistent with the results in the study. At present, selective serotonin reuptake inhibitor (SSRI) drugs such as fluoxetine were used in treating depression, which could increase the concentration of 5-HT in the synaptic cleft to exert antidepressant-like effects. However, the SSRI drugs also had some limitations in the treatment of depression. If the tryptophan could not be replenished in time, it caused the deficiency of 5-HT in the presynaptic membrane and then produced a rapid resistant reaction

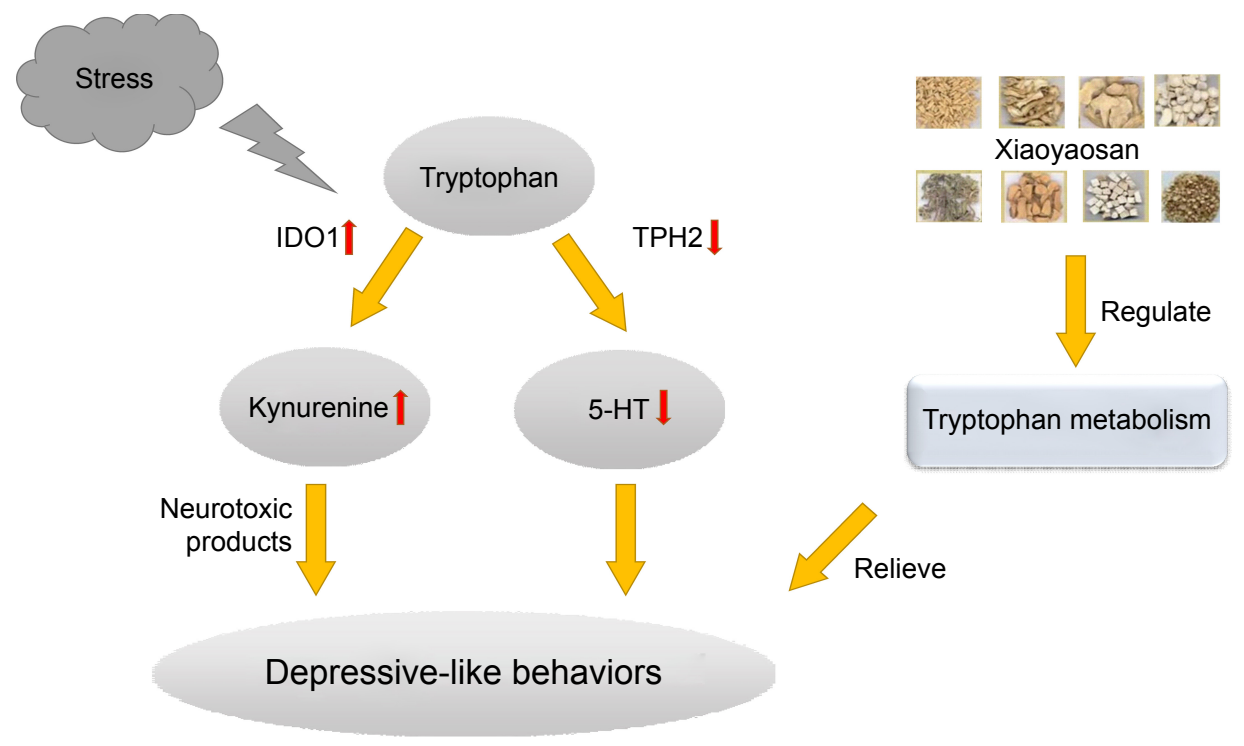

Figure 5 Effects of Xiaoyaosan on CIS-induced changes in hippocampal tryptophan metabolism.

Notes: The behaviors of chronic stress-depressed rats were obviously improved by Xiaoyaosan treatment. The contents of tryptophan and 5-HT and the protein and mRNA levels of TPH2 and IDOI in the hippocampus were influenced by the CIS; these irregular changes could be effectively reversed by the treatment with Xiaoyaosan. Abbreviations: CIS, chronic immobilization stress; 5-HT, 5-hydroxytryptamine; IDOI, indoleamine 2,3-dioxygenase I; TPH2, tryptophan hydrogenase 2. 
and weakened the curative effect. ${ }^{57}$ In this study, there is no report that Xiaoyaosan had such side effects as SSRI drugs, and the antidepressant mechanism of Xiaoyaosan was gradually revealed. We concluded from the present results that Xiaoyaosan had beneficial effects on a CIS rat model through regulating the expressions of THP2 and IDO1 and then affecting tryptophan metabolism and 5-HT system.

However, there still existed several limitations in this study. First, the TPH2 mRNA level in the two treatment groups was different from the control group, which was inconsistent with the results of protein expression, and hence an expanded sample size might avoid this problem. Second, this study only detected the 5-HT-related tryptophan metabolism in hippocampus, whereas amygdalae or raphe nuclei might be potential research points to expound the regulative mechanism of Xiaoyaosan. Third, further studies are needed to examine the effective ingredients of Xiaoyaosan, as well as the specific role of Xiaoyaosan in the regulation of the tryptophan metabolism. For future studies, it is necessary to explore the related brain regions and substances of the 5-HT system, then seek the relationship between the antidepressant-like effect of Xiaoyaosan and the monoamine neurotransmitter system, which will help to determine the pathogenesis of depression and provide a comprehensive basis for the application of Xiaoyaosan.

\section{Conclusion}

In this study, a rat model of depression was successfully established by 21-day CIS. The study proved that Xiaoyaosan played an antidepressant-like effect by regulating the levels of 5-HT, tryptophan, TPH2, and IDO1 in the tryptophan metabolism. As a classic Chinese formula with comprehensive efficacy, Xiaoyaosan had a wide range of applications and could treat mental diseases such as depression and anxiety, and it was worth popularizing in clinical treatment.

\section{Acknowledgment}

This research was supported by grants from National Natural Science Foundation of China (no 81473597, 81630104).

\section{Disclosure}

The authors report no conflicts of interest in this work.

\section{References}

1. Phillips MR, Zhang J, Shi Q, et al. Prevalence, treatment, and associated disability of mental disorders in four provinces in China during 2001-2005: an epidemiological survey. Lancet. 2009;373(9680):2041-2053.

2. Mathers C, Fat DM, Boerma JT. The Global Burden of Disease: 2004 Update. Geneva, Switzerland: World Health Organization; 2008.
3. Yao Y, Huang HY, Yang YX, Guo JY. Cinnamic aldehyde treatment alleviates chronic unexpected stress-induced depressive-like behaviors via targeting cyclooxygenase-2 in mid-aged rats. J Ethnopharmacol. 2015;162:97-103.

4. Carpenter LL, Moreno FA, Kling MA, et al. Effect of vagus nerve stimulation on cerebrospinal fluid monoamine metabolites, norepinephrine, and gamma-aminobutyric acid concentrations in depressed patients. Biol Psychiatry. 2004;56(6):418-426.

5. Castrén E. Is mood chemistry? Nat Rev Neurosci. 2005;6(3):241-246.

6. Cleare AJ, Mcgregor A, O'Keane V. Neuroendocrine evidence for an association between hypothyroidism, reduced central 5-HT activity and depression. Clin Endocrinol. 1995;43(6):713-719.

7. Zill P, Baghai TC, Zwanzger P, et al. SNP and haplotype analysis of a novel tryptophan hydroxylase isoform (TPH2) gene provide evidence for association with major depression. Mol Psychiatry. 2004;9(11): $1030-1036$

8. Gizatullin R, Zaboli G, Jönsson EG, Asberg M, Leopardi R. Haplotype analysis reveals tryptophan hydroxylase (TPH) 1 gene variants associated with major depression. Biol Psychiatry. 2006;59(4):295-300.

9. Harvey M, Shink E, Tremblay M, et al. Support for the involvement of TPH2 gene in affective disorders. Mol Psychiatry. 2004;9(11):980-981.

10. Ara I, Bano S. Citalopram decreases tryptophan 2,3-dioxygenase activity and brain 5-HT turnover in swim stressed rats. Pharmacol Rep. 2012; 64(3):558-566.

11. Eiland L, Mcewen BS. Early life stress followed by subsequent adult chronic stress potentiates anxiety and blunts hippocampal structural remodeling. Hippocampus. 2012;22(1):82-91.

12. Joëls M, Karst H, Alfarez D, et al. Effects of chronic stress on structure and cell function in rat hippocampus and hypothalamus. Stress. 2004; 7(4):221-231.

13. Dang $\mathrm{S}, \mathrm{Lu} \mathrm{Y}, \mathrm{Su} \mathrm{Q}$, et al. H3K9 acetylation of Tph2 involved in depression-like behavior in male, but not female, juvenile offspring rat induced by prenatal stress. Neuroscience. 2018;381:138-148.

14. Lam CS, Tipoe GL, Wong JK, Youdim MB, Fung ML. M30 Antagonizes Indoleamine 2,3-Dioxygenase Activation and Neurodegeneration Induced by Corticosterone in the Hippocampus. PLoS One. 2016; 11(11): 0166966.

15. Meng ZZ, Hu JH, Chen JX, Yue GX. Xiaoyaosan decoction, a traditional Chinese medicine, inhibits oxidative-stress-induced hippocampus neuron apoptosis in vitro. Evid Based Complement Alternat Med. 2012; 2012:489254.

16. Chen JX, Li W, Zhao X, Yang JX, Wei L, Xin Z. Effects of the Chinese traditional prescription Xiaoyaosan decoction on chronic immobilization stress-induced changes in behavior and brain BDNF, TrkB, and NT-3 in rats. Cell Mol Neurobiol. 2008;28(5):745-755.

17. Squire LR. Memory and the hippocampus: a synthesis from findings with rats, monkeys, and humans. PSYCHOL REV. 1992;99:195-231.

18. Zhao HB, Jiang YM, Li XJ, et al. Xiao Yao San Improves the AnxietyLike Behaviors of Rats Induced by Chronic Immobilization Stress: The Involvement of the JNK Signaling Pathway in the Hippocampus. Biol Pharm Bull. 2017;40(2):187-194.

19. Sung-Woo P, Young-Koung K, Jung-Goo L, et al. Antidepressant-like effects of the traditional Chinese medicine kami-shoyo-san in rats. Psychiatry \& Clinical Neurosciences. 2010;61:401-406.

20. Wang R, Xu Y, Wu HL, et al. The antidepressant effects of curcumin in the forced swimming test involve 5-HT1 and 5-HT2 receptors. Eur J Pharmacol. 2008;578(1):43-50.

21. Wang SX, Chen JX, Yue GX, Bai MH, Kou MJ, Jin ZY. Xiaoyaosan decoction regulates changes in neuropeptide y and leptin receptor in the rat arcuate nucleus after chronic immobilization stress. Evid Based Complement Alternat Med. 2012;2012:381278.

22. Li N, Liu Q, Li XJ, et al. TCM Formula Xiaoyaosan Decoction Improves Depressive-Like Behaviors in Rats with Type 2 Diabetes. Evid Based Complement Alternat Med. 2015;2015:415243.

23. Guo X, Qiu W, Liu Y, Zhang Y, Zhao H, Chen J. Effects of Refined Xiaoyaosan on Depressive-Like Behaviors in Rats with Chronic Unpredictable Mild Stress through Neurosteroids, Their Synthesis and Metabolic Enzymes. Molecules. 2017;22(8):1386. 
24. Li XJ, Ma QY, Jiang YM, et al. Xiaoyaosan exerts anxiolytic-like effects by down-regulating the TNF- $\alpha / J A K 2-S T A T 3$ pathway in the rat hippocampus. Sci Rep. 2017;7(1):353.

25. Wu LL, Liu Y, Yan C, Pan Y, Su JF, Wu WK. Antidepressant-Like Effects of Fractions Prepared from Danzhi-Xiaoyao-San Decoction in Rats with Chronic Unpredictable Mild Stress: Effects on HypothalamicPituitary-Adrenal Axis, Arginine Vasopressin, and Neurotransmitters. Evid Based Complement Alternat Med. 2016;2016:1-11.

26. Samarghandian S, Azimi-Nezhad M, Farkhondeh T, et al. Anti-oxidative effects of curcumin on immobilization-induced oxidative stress in rat brain, liver and kidney. Biomedicine \& Pharmacotherapy. 2017; 87:223-229

27. Shin YK, Hsieh YS, Kwon S, Lee HS, Seol GH. Linalyl acetate restores endothelial dysfunction and hemodynamic alterations in diabetic rats exposed to chronic immobilization stress. J Appl Physiol. 2018;124(5): $1274-1283$.

28. Joo Y, Choi KM, Lee YH, et al. Chronic immobilization stress induces anxiety- and depression-like behaviors and decreases transthyretin in the mouse cortex. Neurosci Lett. 2009;461(2):121-125.

29. Sengupta T, Das R, Chattarji S. Chronic but not acute immobilization stress stably enhances hippocampal CA1 metabotropic glutamate receptor dependent Long-Term Depression. Neuroscience Letters. 2016; 633:101-105.

30. Wittenborn AK, Rahmandad H, Rick J, Hosseinichimeh N. Depression as a systemic syndrome: mapping the feedback loops of major depressive disorder. Psychol Med. 2016;46(3):551-562.

31. Ito H, Kawashima R, Awata S, et al. Hypoperfusion in the limbic system and prefrontal cortex in depression: SPECT with anatomic standardization technique. J Nucl Med. 1996;37(3):410-414.

32. Joca SR, Padovan CM, Guimarães FS. [Stress, depression and the hippocampus]. Braz J Psychiatr. 2003;25(Suppl 2):46-51. Portuguese.

33. Kendler KS, Karkowski LM, Prescott CA. The assessment of dependence in the study of stressful life events: validation using a twin design. Psychol Med. 1999;29(6):1455-1460.

34. Willner P, Towell A, Sampson D, Sophokleous S, Muscat R. Reduction of sucrose preference by chronic unpredictable mild stress, and its restoration by a tricyclic antidepressant. Psychopharmacology. 1987; 93(3):358-364.

35. Pothion S, Bizot JC, Trovero F, Belzung C. Strain differences in sucrose preference and in the consequences of unpredictable chronic mild stress. Behav Brain Res. 2004;155(1):135-146.

36. Shalom G, Lifschytz T, Lerer B, Newman ME. Effects of T3 and fluoxetine in the novelty-suppressed feeding test model of depression and anxiety: gender differences. Eur Neuropsychopharmacol. 2006;16:S48-S49.

37. Hallam KT, Horgan JE, Mcgrath C, Norman TR. An investigation of the effect of tacrine and physostigmine on spatial working memory deficits in the olfactory bulbectomised rat. Behav Brain Res. 2004;153(2):481-486.

38. Kuniishi H, Ichisaka S, Yamamoto M, et al. Early deprivation increases high-leaning behavior, a novel anxiety-like behavior, in the open field test in rats. Neurosci Res. 2017;123:27-35.

39. Jing LL, Zhu XX, Lv ZP, Sun XG. Effect of Xiaoyaosan on major depressive disorder. Chin Med. 2015;10:18.

40. Liu XJ, Zhou YZ, Li ZF, et al. Anti-depressant effects of Xiaoyaosan on rat model of chronic unpredictable mild stress: a plasma metabonomics study based on NMR spectroscopy. J Pharm Pharmacol. 2012; 64(4):578-588.
41. Licinio-Paixao J. Hyperinsulinemia; a mediator of decreased food intake and weight loss in anorexia nervosa and major depression. Med Hypotheses. 1989;28(2):125-130.

42. Faulconbridge LF, Wadden TA, Berkowitz RI, et al. Changes in symptoms of depression with weight loss: results of a randomized trial. Obesity. 2009;17(5):1009-1016.

43. Ding XF, Zhao XH, Tao Y, et al. Xiao Yao San Improves DepressiveLike Behaviors in Rats with Chronic Immobilization Stress through Modulation of Locus Coeruleus-Norepinephrine System. Evid Based Complement Alternat Med. 2014;2014:605914.

44. Delgado PL, Moreno FA. Role of norepinephrine in depression. J Clin Psychiat. 2000;61(Suppl 1):5.

45. Varea V, de Carpi JM, Puig C, et al. Malabsorption of carbohydrates and depression in children and adolescents. J Pediatr Gastroenterol Nutr. 2005;40(5):561-565.

46. Walther DJ, Peter JU, Bashammakh S, et al. Synthesis of serotonin by a second tryptophan hydroxylase isoform. Science. 2003;299(5603):76.

47. Abkevich V, Camp NJ, Hensel CH, et al. Predisposition locus for major depression at chromosome 12q22-12q23.2. Am J Hum Genet. 2003; 73(6):1271-1281.

48. Hvas AM, Juul S, Bech P, Nexø E. Vitamin B6 level is associated with symptoms of depression. Psychother Psychosom. 2004;73(6): 340-343.

49. Maiwald S, Wehner R, Schmitz M, Bornhäuser M, Loeb S, Wassmuth R. IDO1 and IDO2 gene expression analysis by quantitative polymerase chain reaction. Tissue Antigens. 2011;77(2):136-142.

50. Elovainio M, Hurme M, Jokela M, et al. Indoleamine 2,3-dioxygenase activation and depressive symptoms: results from the Young Finns Study. Psychosom Med. 2012;74(7):675-681.

51. Murakami Y, Ishibashi T, Tomita E, et al. Depressive symptoms as a side effect of Interferon- $\alpha$ therapy induced by induction of indoleamine 2,3-dioxygenase 1. Sci Rep. 2016;6:29920.

52. Jiang X, Xu L, Tang L, et al. Role of the indoleamine-2,3-dioxygenase/ kynurenine pathway of tryptophan metabolism in behavioral alterations in a hepatic encephalopathy rat model. J Neuroinflammation. 2018;15(1):3.

53. Clarke G, Fitzgerald P, Cryan JF, Quigley EM, Dinan TG. S1804 Altered Kynurenine Pathway Metabolism in Irritable Bowel Syndrome (IBS)-Evidence of Indoleamine 2,3-Dioxygenase (IDO) Activation in a Male Cohort. Gastroenterology. 2008;134(4):273-270.

54. Wang J, Li X, He S, et al. Regulation of the kynurenine metabolism pathway by Xiaoyao San and the underlying effect in the hippocampus of the depressed rat. J Ethnopharmacol. 2018;214:13-21.

55. Shishkina GT, Kalinina TS, Dygalo NN. Up-regulation of tryptophan hydroxylase- 2 mRNA in the rat brain by chronic fluoxetine treatment correlates with its antidepressant effect. Neuroscience. 2007;150(2): 404-412.

56. Yang JM, Rui BB, Chen C, et al. Acetylsalicylic acid enhances the anti-inflammatory effect of fluoxetine through inhibition of NF- $\mathrm{B}$, p38-MAPK and ERK1/2 activation in lipopolysaccharide-induced BV-2 microglia cells. Neuroscience. 2014;275:296-304.

57. Oxenkrug GF. Metabolic syndrome, age-associated neuroendocrine disorders, and dysregulation of tryptophan-kynurenine metabolism. Ann N Y Acad Sci. 2010;1199:1-14
Neuropsychiatric Disease and Treatment

\section{Publish your work in this journal}

Neuropsychiatric Disease and Treatment is an international, peerreviewed journal of clinical therapeutics and pharmacology focusing on concise rapid reporting of clinical or pre-clinical studies on a range of neuropsychiatric and neurological disorders. This journa is indexed on PubMed Central, the 'PsycINFO' database and CAS,

\section{Dovepress}

and is the official journal of The International Neuropsychiatric Association (INA). The manuscript management system is completely online and includes a very quick and fair peer-review system, which is all easy to use. Visit http://www.dovepress.com/testimonials.php to read real quotes from published authors. 